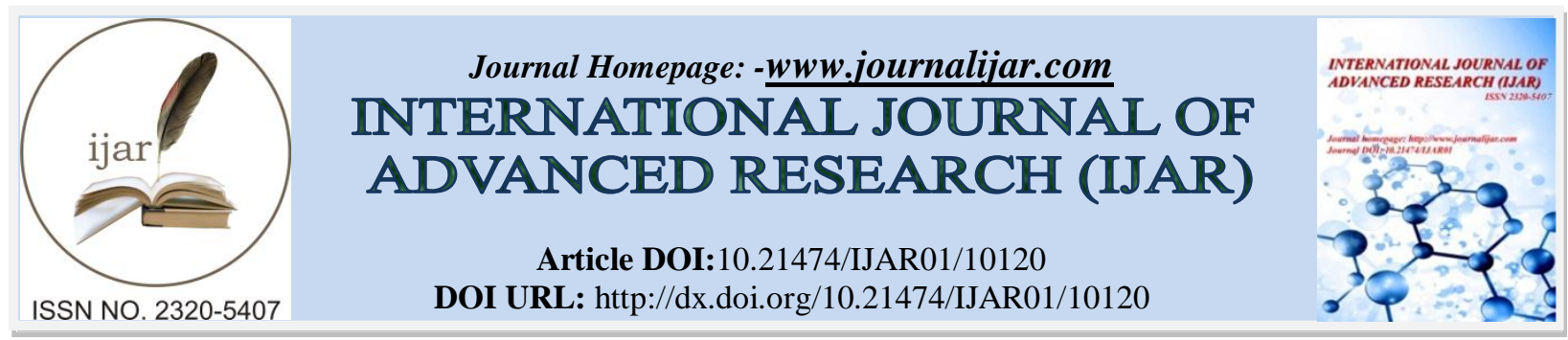

RESEARCH ARTICLE

\title{
ASSESS THE KNOWLEDGE AND PERCEPTION ON HUMAN BREAST MILK BANK AMONG POSTNATAL MOTHERS.
}

Sharumathy $\mathbf{V}^{1}$, Dr.Vetriselvi. $P^{2}$ and Dr.Adhisivam. $B^{3}$.

1. B.SC[N],College of nursing,JIPMER,Puducherry.

2. Assistant professor,College of Nursing,JIPMER,Puducherry.

3. Additional Professor\&HOD,Department of neonatalogy,JIPMER, Puducherry.

\section{Manuscript Info}

\section{Manuscript History}

Received: 01 October 2019

Final Accepted: 03 November 2019

Published: December 2019

Key words:-

Human breastmilk bank, Knowledge,

Perception, Postnatal Mothers.

\section{Abstract}

Background:Human breast milk is considered as the best source of all nutrients, beyond that it the only living food source for infants especially during the first six months of their life. WHOrecommendstheexclusivebreastfeedingtill6monthsoflifeforallinfan tsandthensupplemented breastfeedingisrecommended untilat least two yearsofageanditisrecognized asgloballyhighlymore Nutritious. According to the joint statement given by the World Health Organization and United Nations InternationalChildren'sEmergency Fund(UNICEF)in1980. Thebestandessentialfoodforany baby whoseown mother'smilkisnotavailable

isthebreastmilkfromanotherdonatinghealthymother. Hence this study focuseson identifying andassessing theknowledgeand perceptionamong postnatalmothers aboutHumanBreastMilk Bank establishmentand itsdonation.Itaimsondescribing

behaviors,beliefs, andfeelingsbehindHumanMilk Bank anditsdonation by identifying donor's individual,socialandenvironmentalfeatures thatcouldaffectthedonationofhumanmilk. Thefindings willhelp todevelophealtheducation toolsto promotehumanmilkdonation by identifying the factors that hinders the human milk donation and rectifying it accordingly.

Objectives:

1. Toassessknowledgeand perception on Human MilkBankamongpostnatal mothers.

2. Toidentifytheassociationofknowledgeand perception withselected demographic variables.

Methods: The design of the study is cross sectional. Theparticipantswho met the inclusion criteriawereselected byconvenientsamplingtechnique. The total participants included in this study were 100 . the data was collected by using structured questionnaire. Statistical methods like frequency and percentage, chisquare test have been used and All statistical analysis has been carried out at $5 \%$ level of significant and $\mathrm{P}<0.05$ has considered as significant.

Results:The study concludedthatonly 19\%ofthepostnatalmothers hadadequate knowledgeandinregardto

Corresponding Author:- Sharumathy V. 
perception $70 \%$ ofpostnatalmothershadfavorable perception. Therewasno significantassociation

ofknowledgeandperception with any of thesocio- demographic variables.

Conclusion:The above results stress the importance of organizing health education topostnatalmothers.

Copy Right, IJAR, 2019,. All rights reserved.

\section{Introduction:-}

Human breast milk is considered as the best source of all nutrients, beyond that it is the only living food source for infants especially during the first six months of their life. ${ }^{(1)}$

WHOrecommendstheexclusivebreastfeedingtill6monthsoflifeforallinfantsandthensupplementedbreastfeedingisreco mmended untilat least two yearsofageanditisrecognized asgloballyhighlymore Nutritious. ${ }^{(2)}$

It is vital because of its bio-active substances like free amino acids, nucleotides, cells, growth factors, enzymes, hormones, pro-biotic bacteria, and prebiotic oligosaccharides that are essential for development of immature immune system and gut micro biome of both full-term and high risk newborns like low birth weight and preterm. It also decreases the incidences of necrotizing enterocolitis , late onset sepsis, retinopathy, food intolerance or malabsorption, immunodeficiency and improvise the neuro-cognitive development. Meanwhile lower the risk of childhood diabetes, obesity compared to those infants fed with formula milk and animal's milk. ${ }^{(1,3,4,10,12)}$

Exclusivebreastfeedingisveryessential not only for infants but also for mothers as it prevents the risk of breast cancer and enhances the bond between mothers and babies. ${ }^{(11)}$

According to the joint statement given by the World Health Organization and United Nations InternationalChildren'sEmergency Fund(UNICEF)in1980. Thebestandessentialfoodforany baby whoseown mother'smilkisnotavailable isthebreastmilkfromanotherdonatinghealthymother ${ }^{(5)}$.

BreastMilkDonation isrecommendedandsuggestedforhospitalized (sick)orpreterm babiesinwhich theirmothersdon'thavesufficientmilk production (or) underwent any breast surgery in past (or) under any medications like chemotherapy (or) any otherconditionwheremothercan'tbeableto giveherbreastmilk. The Human milk bank receives human milk from donor after screening, then collects, process, pasteurize, store and then distributed to needy. ${ }^{(6,7,9,12)}$

But unfortunately, most of the developing countries including our nation which has highest burden of global neonatal mortality and morbidity rate lag in the implementation of donor breastmilk as best alternative choice for vulnerable infants. Breast milk banks aren't a new phenomenon in India, but they are scarce. Eventhough Asia's first milk bank was set up in Mumbai (Lokmanya Tilak Hospital) in 1989. Currently there are only 60 human milk bank. Whereas Brazil, another developing country, is an international leader with 217 banks and 113 collection centers which has helped to reduce brazil's infant mortality rate by $73 \% .^{(1,3,8,9)}$

Hence this study focuseson identifying andassessing theknowledgeand perceptionamong postnatalmothers aboutHumanBreastMilk Bank establishmentand itsdonation.Itaimsondescribing behaviors, beliefs, andfeelingsbehindHumanMilk Bank anditsdonation by identifying donor's individual,socialandenvironmentalfeatures thatcouldaffectthedonationofhumanmilk. Thefindings willhelp todevelophealtheducation toolsto promotehumanmilkdonation by identifying the factors that hinders the human milk donation and rectifying it accordingly.

\section{Objectives:-}

1. Toassessknowledgeand perception on Human MilkBankamongpostnatal mothers.

2. Toidentifytheassociationofknowledgeand perception withselected demographic variables. 
Methodology:-

Study design:

Cross sectional study

Inclusion criteria:

Postnatalmothers inWomen and Children HospitalJIPMER

Exclusion criteria:

Motherswho aresickduringthedatacollection procedure

\section{Sampling population:}

Postnatal mothers who are admitted in Women and ChildrenHospitalJIPMER .

\section{Sample size: 100}

\section{Sampling technique:}

Convenience sampling technique.

\section{Tool description:}

structured questionnairewasused tocollectdatafrom postnatal mothers.

\section{TheQuestionnairehad 3sections. Theyare:}

Section - A: Socio-Demographic Data

Section-B: Knowledge on human breast milk bank.

Section-C: Perception on human breast milk bank.

\section{Study procedure:}

Thestudy wasconductedafterapproval fromIEC(Humanstudies), JIPMER. Theparticipantswho met the inclusion criteriawereselected byconvenientsamplingtechnique. The total participants included in this study were 100 .

Aftergettinginformedwrittenconsentfrom the postnatal mothers, a structuredquestionnaire was administered to them to assess their knowledge and perception about human breast milk bank. In which it had 16 knowledge questions [multiple choicequestions ]and 13 perception questions and instructed themto markthecorrectanswer.

Inknowledgequestionnairethecorrectanswerwasscoredas1andthewronganswer as0.Inperceptionquestionnaire,theresponseoptionsforeachquestionwasstrongly agree,agree, not sure,disagreeand stronglydisagree.

\section{Parameters studied:}

Independent variable:

1. Age

2. Education

3. Religion

4. Type of family

5. Monthly income of family

6. Domicile

\section{Outcome variables:}

Level ofKnowledge and perception on human milk bank.

\section{Confounding and interacting variables:}

NIL

\section{Statistical test used for data analysis:}

Thedistributions of socio-demographic characteristics were expressedasfrequency andpercentage. The knowledgelevelwascategorizedintoinadequate,moderateandadequatebased onthescoresanditwas expressedin 
frequency andpercentage.Perception levelwascategorizedinto unfavorable, averageand favorablebasedonthescoresanditwasexpressedinfrequencyandpercentage.Theassociationoflevel

ofknowledge\&perceptioncategorieswithdemographicvariableswascarriedoutbyusingchi-square test. All statistical analysis has been carried out at $5 \%$ level of significant and $\mathrm{P}<0.05$ has considered as significant.

Results:-

Table1:-Distribution of socio-demographicvariables of postnatal mothers $\quad \mathbf{N}=\mathbf{1 0 0}$

\begin{tabular}{|c|c|c|c|}
\hline Variable & Category & Frequency(N) & Percentage $(\%)$ \\
\hline \multirow[t]{2}{*}{ Agein years } & $20-30$ & 90 & $90 \%$ \\
\hline & $31-40$ & 10 & $10 \%$ \\
\hline \multirow[t]{3}{*}{ Domicile } & Rural & 73 & $73 \%$ \\
\hline & Semi-urban & 0 & $0 \%$ \\
\hline & Urban & 27 & $27 \%$ \\
\hline \multirow[t]{4}{*}{ Education } & Illiterate & 23 & $23 \%$ \\
\hline & $6^{\text {th }}-10^{\text {th }}$ & 20 & $20 \%$ \\
\hline & $11^{\text {th }}-12^{\text {th }}$ & 25 & $25 \%$ \\
\hline & Graduate & 32 & $32 \%$ \\
\hline \multirow[t]{3}{*}{ Type of family } & Nuclear & 36 & $36 \%$ \\
\hline & Joint & 64 & $64 \%$ \\
\hline & Extended & 0 & $0 \%$ \\
\hline \multirow{4}{*}{$\begin{array}{l}\text { Monthly income } \\
\text { of family }\end{array}$} & $\leq 2000$ & 32 & $32 \%$ \\
\hline & $2001-5000$ & 19 & $19 \%$ \\
\hline & $5001-10,000$ & 21 & $21 \%$ \\
\hline & $>10,000$ & 28 & $28 \%$ \\
\hline
\end{tabular}

Table 2:-Knowledgelevel of postnatal mothers: $\mathbf{N = 1 0 0}$

\begin{tabular}{|l|l|l|l|}
\hline Groups & Knowledge score & Frequency(NO) & Percentage(\%) \\
\hline Postnatal & Inadequate $(0-50 \%)$ & 29 & $29 \%$ \\
mothers & Moderate $(51-75 \%)$ & 52 & $52 \%$ \\
& Adequate $(>75 \%)$ & 19 & $19 \%$ \\
\hline
\end{tabular}

Table 3:-Perception levelof postnatal mothers:

$\mathbf{N}=\mathbf{1 0 0}$

\begin{tabular}{|l|l|l|l|}
\hline Groups & Perceptionscore & Frequency(NO) & Percentage(\%) \\
\hline Postnatal & Unfavorable (0-50\%) & 1 & $1 \%$ \\
mothers & Average (51-75\%) & 29 & $29 \%$ \\
& Favorable (75-100\%) & 70 & $70 \%$ \\
\hline
\end{tabular}

Table 4:-Association between knowledge and perception of postnatal mothers with socio demographic variables: $\mathrm{N}=\mathbf{1 0 0}$

\begin{tabular}{|c|c|c|c|c|c|c|c|c|c|c|}
\hline \multirow{2}{*}{$\begin{array}{l}\text { S } \\
\text { I. } \\
\text { N } \\
\text { o }\end{array}$} & \multirow[t]{2}{*}{ Variables } & \multirow[t]{2}{*}{$\mathbf{N}$} & \multicolumn{3}{|c|}{ Knowledgescore } & \multirow{2}{*}{$\begin{array}{l}\mathbf{P} \\
\text { valu } \\
\mathrm{e}\end{array}$} & \multicolumn{3}{|c|}{ Perceptionscore } & \multirow{2}{*}{$\begin{array}{l}P \\
\text { val } \\
\text { ue }\end{array}$} \\
\hline & & & $\begin{array}{l}\text { inadequ } \\
\text { ate }\end{array}$ & $\begin{array}{l}\text { moder } \\
\text { ate }\end{array}$ & $\begin{array}{l}\text { adequ } \\
\text { ate }\end{array}$ & & $\begin{array}{l}\text { unfavora } \\
\text { ble }\end{array}$ & $\begin{array}{l}\text { avera } \\
\text { ge }\end{array}$ & $\begin{array}{l}\text { Favora } \\
\text { ble }\end{array}$ & \\
\hline 1 & $\begin{array}{l}\text { Agein years } \\
20-30 \\
31-40\end{array}$ & $\begin{array}{l}9 \\
0 \\
1 \\
0\end{array}$ & $\begin{array}{l}26 \\
3\end{array}$ & $\begin{array}{l}45 \\
7\end{array}$ & $\begin{array}{l}19 \\
0\end{array}$ & $\begin{array}{l}0.24 \\
6\end{array}$ & $\begin{array}{l}1 \\
0\end{array}$ & $\begin{array}{l}27 \\
2\end{array}$ & $\begin{array}{l}62 \\
8\end{array}$ & $\begin{array}{l}0.7 \\
48\end{array}$ \\
\hline 2 & $\begin{array}{l}\text { Domicile } \\
\text { Rural } \\
\text { Semi -urban } \\
\text { Urban }\end{array}$ & $\begin{array}{l}7 \\
3 \\
0 \\
2 \\
\end{array}$ & $\begin{array}{l}23 \\
0 \\
6\end{array}$ & $\begin{array}{l}36 \\
0 \\
16\end{array}$ & $\begin{array}{l}14 \\
0 \\
5\end{array}$ & $\begin{array}{l}0.06 \\
17\end{array}$ & $\begin{array}{l}1 \\
0 \\
0\end{array}$ & $\begin{array}{l}19 \\
0 \\
10\end{array}$ & $\begin{array}{l}53 \\
0 \\
17\end{array}$ & $\begin{array}{l}0.4 \\
83\end{array}$ \\
\hline
\end{tabular}




\begin{tabular}{|c|c|c|c|c|c|c|c|c|c|c|}
\hline & & 7 & & & & & & & & \\
\hline 3 & $\begin{array}{l}\text { Education } \\
\text { Illiterate } \\
6^{\text {th }}-10^{\text {th }} \\
11^{\text {th }}-12^{\text {th }} \\
\text { Graduate }\end{array}$ & $\begin{array}{l}2 \\
3 \\
2 \\
0 \\
2 \\
5 \\
3 \\
2\end{array}$ & $\begin{array}{l}9 \\
6 \\
8 \\
6\end{array}$ & $\begin{array}{l}9 \\
11 \\
13 \\
19\end{array}$ & $\begin{array}{l}5 \\
3 \\
4 \\
7\end{array}$ & $\begin{array}{l}0.72 \\
0\end{array}$ & $\begin{array}{l}1 \\
0 \\
0 \\
0\end{array}$ & $\begin{array}{l}7 \\
3 \\
9 \\
10\end{array}$ & $\begin{array}{l}15 \\
17 \\
16 \\
22\end{array}$ & $\begin{array}{l}0.41 \\
8\end{array}$ \\
\hline 4 & $\begin{array}{l}\text { Typeoffamily } \\
\text { Nucl } \\
\text { ear } \\
\text { Joint } \\
\text { Exte } \\
\text { nded }\end{array}$ & $\begin{array}{l}3 \\
6 \\
6 \\
4 \\
0\end{array}$ & $\begin{array}{l}9 \\
20 \\
0\end{array}$ & $\begin{array}{l}20 \\
32 \\
0\end{array}$ & $\begin{array}{l}7 \\
12 \\
0\end{array}$ & $\begin{array}{l}0.79 \\
7\end{array}$ & $\begin{array}{l}1 \\
0 \\
0\end{array}$ & $\begin{array}{l}6 \\
23 \\
0\end{array}$ & $\begin{array}{l}29 \\
41 \\
0\end{array}$ & $\begin{array}{l}0.06 \\
0\end{array}$ \\
\hline 5 & $\begin{array}{l}\text { Monthlyinco } \\
\text { me } \\
\text { offamily } \\
\leq 2000 \\
2001-5000 \\
5000-10,000 \\
>10,000\end{array}$ & $\begin{array}{l}3 \\
2 \\
1 \\
9 \\
2 \\
1 \\
2 \\
8\end{array}$ & $\begin{array}{l}12 \\
5 \\
6 \\
6\end{array}$ & $\begin{array}{l}15 \\
9 \\
10 \\
18\end{array}$ & $\begin{array}{l}5 \\
5 \\
5 \\
4\end{array}$ & $\begin{array}{l}0.69 \\
8\end{array}$ & $\begin{array}{l}0 \\
0 \\
1 \\
0\end{array}$ & $\begin{array}{l}10 \\
4 \\
7 \\
8\end{array}$ & $\begin{array}{l}22 \\
15 \\
13 \\
20\end{array}$ & $\begin{array}{l}0.57 \\
0\end{array}$ \\
\hline
\end{tabular}

\section{Discussion:-}

ThefirstobjectiveofthestudywastoassesstheknowledgeandperceptiononHumanMilk Bank among postnatal mothers.

Among postnatalmothers only 19\%hadadequateknowledge,52\%hadmoderateknowledgeand29\% of them hadinadequateknowledgeandInregardtoperception70\%ofthepostnatalmothershadfavorable perception, $29 \%$ ofthemhad averageperceptionand $1 \%$ hadunfavorableperception.

\section{Theabovefindingsweresupported by thefollowingstudies:}

GoodfellowHE, Reimers P,Ballard K,Coutsoudis $\mathrm{A}(2016)^{(13)}$ conducted astudy insouthAfrica to determinethemothersperceptionofbreastfeedingandcommunity-basedhumanmilkbanks.Through conveniencesamplingatotalof40motherswereinterviewed.Theresultsshowedthatonly58\% of womenwerewillingtodonatethebreastmilk,only30\% werewillingtofeedtheirbabywithdonated milkand only $57.5 \%$ consideredthe ideaofsettingupcommunitybased human milkbank.

Leung J,YauS(2015) ${ }^{(6)}$ conductedastudy inHong Kongtoexploretheperceptionsonbreastmilk donationandestablishmentofbreastmilkbankamongbreastfeedingmothers. Aqualitativedescriptive studywasadopted.Throughpurposivesampling10participantswereselected. Thedatawerecollected throughin-depth face-to-face andsemi-structuredinterviews. The resultsshowedthatmostof the breastfeedingmotherswelcomedtheideaofbreastmilkdonationandtheestablishmentofbreastmilk bank.

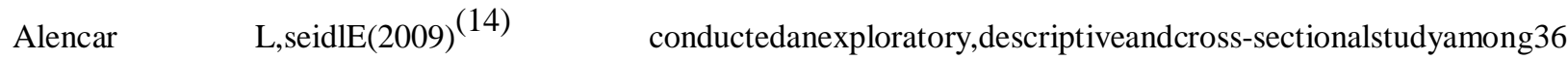
womenintheagegroupof 14to33yearswho were breastmilk donorsinBrazil. Theobjectivewas to describethecharacteristicsofdonationbehaviorandtoidentifythereasons, beliefsandfeelingsrelative tobreastmilk donationpractice. Throughconvenientsampling thesampleswereselectedanddatawere collectedthroughinterviewmethod.Theresultsshowedthat $91.7 \%$ ofthemothersthoughtthatbreast milkdonationexperience ispositive.Themostfrequently reportedreasonsfordonatingbreastmilkwere altruismand excessmilkproduction.

Iloh K, Osuorah C, Ndu I, et.all. $(2018)^{(1)}$ conducted a study in south-east Nigeria to determine the perception and acceptability of donor breast milk among mothers. It is a cross- sectional multi-center study in which the data was collected using pretested questionnaire among 1235 selected mothers by using purposive and convenient sampling 
method. Results showed that 39\% (480/1225) of them heard about it, whereas only $10 \%$ had adequate knowledge about the concept and policy of donor milk and $60 \%$ of them were willing to donate and use the donor breast milk.

\section{Thesecondobjectiveofthestudy wastoidentifytheassociationofknowledgeandperception with selected demographicvariables:}

Thecurrentstudy findingsrevealedthattherewasnosignificantassociationofknowledgeandperception with anyof theselecteddemographicvariablesamong postnatalmothers.

\section{Theabovefindingsweresupported by thefollowingstudies:}

Ghuge S, Aghamkar J, Salvi R (2018) ${ }^{(7)}$ conducted a study in Maharastra to determine the knowledge and attitude towards human milk bank among postnatal mothers. The research design was one group pretest posttest quasi experimental design. Descriptive research approach has been carried out. By using non-probability convenience sampling technique a total of 60 postnatal mothers were included in this study and the data has been collected through structured knowledge and attitude questionnaires. The results concluded that there was no significant association between the baseline variables with knowledge, attitude scores and the demographic variables.

\section{Summary:}

The study concludedthatonly19\%ofthepostnatal mothershadadequate knowledgeandinregardto perception70\%ofpostnatal mothershadfavorable perception. Therewasno significantassociationofknowledgeandperception with any of thesocio- demographic variables.

\section{Conclusion:-}

The above results stress the importance of organizing health education topostnatalmothers, broadcasting postnatalward\&NICUwillenhancetheirknowledgeandincreasethenumberofdonors.Handouts stressingtheimportanceofhumanmilkdonation should beprovidedto allpostnatalmother

\section{References:-}

1. AlencarLC,SeidlEM.Breastmilkdonation:women'sdonorexperience.Rev.SaudePublica.2009;43:70-77.

2. Arnold LDW.Humanmilkbanking in North America. JHumLact.1997;13:159-62.

3. Barin J, Lotscher M. Contextualizing Human Milk Banking and Milk Sharing Practices and Perception in Switzerland. THE MILK GAP report. 2018 (August)

4. DennisCL. Breastfeeding Initiation andDuration.J ObstetGynecol Neonatal Nurs.2001;31:12-32.

5. Donor breast milk banks: the operation of donor milk bank services. NICE clinical guideline 93.2018 (July).

6. Ghuge S, Aghamkar J, Salvi R. A study to assess the knowledge and attitude regarding donating milk to the human milk bank among postnatal mothers of selected hospital. International Journal of Applied Research. 2018; 4(9): 94-98.

7. GoodfellowHE,ReimersP,BallardK,CoutsoudisA.Perceptionsofcommunity-basedhumanmilkbanks beforeand aftertraining ina resource-limited SouthAfrican setting. S Afr J ChildHealth.2016;10(1):83-86.

8. Iloh K, Osuorah C, Ndu I, Asinobi I, Anyim I,et al. Perception of donor breast milk and determinants of its acceptability among mothers in a developing community: a cross-sectional multi-center study in south-east Nigeria. International Breastfeeing Journal . 2018; 13:47 : 1-2.

9. Kim JH, Unger S. Human Milk Banking. Canadian Pediatric society Nutrition and Gastroenterology Committee.Paediatr ChildHealth .2010;15:595-98.

10. Mackenzie C, Hons B, Javanparast S, Newman L,Hons B. Mother's knowledge of and attitudes toward human milk banking in south Australia: A qualitative study. Journal of Human Lactation. 2013; 29(2):222-29.

11. McGuireW,AnthonyMY.Donorhumanmilkversusinfantformulaforpreventingnecrotizingenter colitis inpreterminfants:systematicreview. ArchDisChild Fetal NeonatalEd.2003;88:11-14.

12. WilliamsAF,FisherC,GreasleyV,TraylerH,WoofrideMW.Humanmilkbanking.JTropPediatr.1985; 31:185-90.

13. Wong'sTextbookofEssential pediatricNursing .7thed.Philadelphia:Elsevier publications;2005.

14. YauSY,LeungJCY.PerceptionsofbreastfeedingMothersonBreastMilkDonationandEstablishmentofHuman BreastMilkBankinHong Kong. IntJ NursPract .2015; 2:72-80. 